PROCEEDINGS OF THE

AMERICAN MATHEMATICAL SOCIETY

Volume 139, Number 9, September 2011, Pages 3307-3316

S 0002-9939(2011)10741-X

Article electronically published on February 1, 2011

\title{
KOLMOGOROV COMPLEXITY AND STRONG APPROXIMATION OF BROWNIAN MOTION
}

\author{
BJØRN KJOS-HANSSEN AND TAMÁS SZABADOS
}

(Communicated by Julia Knight)

\begin{abstract}
Brownian motion and scaled and interpolated simple random walk can be jointly embedded in a probability space in such a way that almost surely the $n$-step walk is within a uniform distance $O\left(n^{-1 / 2} \log n\right)$ of the Brownian path for all but finitely many positive integers $n$. Almost surely this $n$-step walk will be incompressible in the sense of Kolmogorov complexity, and all Martin-Löf random paths of Brownian motion have such an incompressible close approximant. This strengthens a result of Asarin, who obtained instead the bound $O\left(n^{-1 / 6} \log n\right)$. The result cannot be improved to $o\left(n^{-1 / 2} \sqrt{\log n}\right)$.
\end{abstract}

\section{INTRODUCTION}

1.1. Algorithmic randomness and probability. Almost sure statements in probability theory usually do not come with examples, but algorithmically random objects, defined in terms of Turing computability theory, have most of the properties expected in almost sure behavior. The value of their study lies in the extent to which all almost sure properties of interest are reflected in each algorithmically random object.

The case of infinite binary sequences with fair-coin measure, equivalently the unit interval $[0,1]$ with Lebesgue measure, is by Carathéodory's measure algebra isomorphism theorem in some sense sufficient 13 . This case has been deeply studied; see for instance the recent book by Nies [16. In the present paper we follow up on work of Asarin and Pokrovskii [2] and Fouché [8] on algorithmic randomness in the context of Brownian motion, i.e. Wiener measure on the space of continuous functions $C[0,1]$. Our goal is to strengthen and "explain" a theorem of Asarin relating Brownian motion and random walks to Kolmogorov complexity. The isomorphism between the measure algebras of $C[0,1]$ and $[0,1]$ does not help here, because the theorem involves metric structure.

1.2. Schnorr and Asarin's theorems. The algorithmic randomness of an infinite object $A$ may often be expressed in terms of the complexity of its finite

Received by the editors February 24, 2009 and, in revised form, April 4, 2009 and August 16, 2010

2010 Mathematics Subject Classification. Primary 68Q30, 03D32; Secondary 60F15.

This material is based upon work supported by the National Science Foundation under Grants No. 0652669 and 0901020. Thanks are due to the anonymous referee for very helpful comments and to Jacob Woolcutt for assistance with the production of Figure 1.

(C)2011 American Mathematical Society

Reverts to public domain 28 years from publication 
approximations. This is most well known in the case of infinite binary strings $A \in 2^{\omega}=\{0,1\}^{\infty}$.

Definition 1.1. Let $\mu$ be the fair-coin measure on $2^{\omega}$. A real $A \in 2^{\omega}$ is Martin-Löf random if for each uniformly $\Sigma_{1}^{0}$ sequence $\left\{U_{n}\right\}_{n \in \mathbb{N}}, U_{n} \subseteq 2^{\omega}$, with $\mu U_{n} \leq 2^{-n}$, we have $A \notin \bigcap_{n} U_{n}$.

Let $K$ denote prefix-free Kolmogorov complexity [15] over either the usual alphabet $\{0,1\}$ or the alternative $\{ \pm 1\}=\{1,-1\}$. The Kolmogorov complexity of an element of $\{ \pm 1\}^{n}$ can be identified with the Kolmogorov complexity of the corresponding string in $\{0,1\}^{n}$ under (say) the map given by $-1 \mapsto 0$ and $1 \mapsto 1$.

Theorem 1.2 (Schnorr). $1 A \in 2^{\omega}$ is Martin-Löf random if and only if there is a constant $c$ such that for all $n, K(A\lceil n) \geq n-c$.

In his dissertation [1, Kolmogorov's student Asarin defined Martin-Löf random Brownian motion analogously to Definition 1.1 and obtained an analogue of Schnorr's Theorem 1.2 which we now describe.

Let $C[0,1]$ denote the space of continuous functions $f:[0,1] \rightarrow \mathbb{R}$ with the uniform metric $d$ given by

$$
d(f, g)=\max _{t \in[0,1]}|f(x)-g(x)|
$$

and with the Wiener measure $\mathbb{P}$ underlying Brownian motion as in Durrett 7 . Let $\mathfrak{S}$ be the set of all balls contained in $C$ with rational radii whose centers are piecewise linear functions with rational break points and rational values at the break points. The set $\mathfrak{S}$ is countable, and we can specify a member of $\mathfrak{S}$ by specifying a finite list of rational numbers. We may write $\mathfrak{S}=\left\{T_{n}: n \in \mathbb{N}\right\}$, where the list of rational numbers representing $T_{n}$ is uniformly computable. For any total computable function $\psi: \mathbb{N} \times \mathbb{N} \rightarrow \mathbb{N}$, the set $U_{n}=\bigcup_{m} T_{\psi(n, m)}$ is called a $\Sigma_{1}^{0}$ subset of $C[0,1]$. A set $M \subseteq C[0,1]$ is a Martin-Löf null set if there is a total computable function $\psi: \mathbb{N} \times \mathbb{N} \rightarrow \mathbb{N}$ such that for $U_{n}=\bigcup_{m} T_{\psi(n, m)}$, we have $\mathbb{P}\left(U_{n}\right) \leq 2^{-n}$ and $M \subseteq \bigcap_{n} U_{n}$.

Theorem 1.3 (Asarin [2]). The union $M_{0}$ of all Martin-Löf null sets is a MartinLöf null set.

A function $x \in C[0,1]$ is called Martin-Löf random if $x \notin M_{0}$. Next, we define the analogue of the other side of Schnorr's Theorem. A sequence of binary strings $\alpha_{n} \in\{ \pm 1\}^{n}, n \in \mathbb{N}$, is said to be complex if there is a constant $c>0$ such that for all $n \in \mathbb{N}$,

$$
K\left(\alpha_{n}\right) \geq n-c .
$$

Let $C_{n}$ be the set of all functions $f \in C[0,1]$ that are linear with slope $\pm \sqrt{n}$ in each interval $\left[\frac{i-1}{n}, \frac{i}{n}\right], 1 \leq i \leq n$, and such that $f(0)=0$. Each function $f \in C_{n}$ is associated with a finite string, where -1 (1) represents an interval where $f$ is decreasing (increasing). If this string is $x$, then we write $f=\ell_{x}$. Thus we can speak of a sequence of functions $f_{n} \in C_{n}, n \in \mathbb{N}$, being complex as well. We are now in a position to state Asarin's result.

\footnotetext{
${ }^{1}$ The result was announced by Chaitin [4] and attributed to Schnorr (who was the referee of the paper) without proof. The first published proof (in a form generalized to arbitrary computable measures) appeared in the work of Gacs 10 .
} 
Theorem 1.4 (Asarin [2]). $W \in C[0,1]$ is a Martin-Löf random Brownian motion if and only if there is a constant $c$ such that for all but finitely many $n \in \mathbb{N}$ there is a string $x=\left(x_{1}, \ldots, x_{n}\right) \in\{ \pm 1\}^{n}$ such that

$$
d\left(\ell_{x}, W\right) \leq n^{-1 / 10} \quad \text { and } \quad K\left(x_{1}, \ldots, x_{n}\right) \geq n-c .
$$

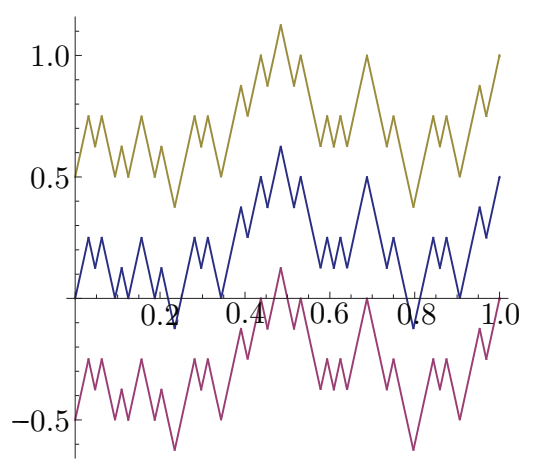

FiguRE 1 . The ball of radius $n^{-1 / 6}$ around $\ell_{x}$ for a randomly chosen $x \in\{ \pm 1\}^{n}$, where $n=4^{3}$.

The bound $n^{-1 / 10}$ was also quoted and used by Fouché 8, 9 . In his dissertation 1], Asarin actually improved the bound to $20 n^{-1 / 6} \log n$; an instance of the bound $n^{-1 / 6}$ is illustrated in Figure 1. In the present paper we will further improve and explain these bounds. In order to do so, we will use strong approximation of Brownian motion, i.e. the joint realization of Brownian motion and random walks on the same probability space in such a way that the Brownian path and a scaled and interpolated random walk with $n$ steps in the unit interval are almost surely within a $d$-distance $O\left(n^{-1 / 2} \log n\right)$. The proof of this fact is based on a finite horizon version of a celebrated embedding theorem by Komlós, Major, and Tusnády [11,12]: Theorem 2.3 .

\section{JOINT EMBEDDING OF RANDOM VARIABLES}

We start with $\left\{X_{i}\right\}_{i \in \mathbb{N}}$, a sequence of independent random variables satisfying

$$
\mathbb{P}\left\{X_{i}=1\right\}=\mathbb{P}\left\{X_{i}=-1\right\}=\frac{1}{2} .
$$

For any $n \geq 0$, let $S_{n}=\sum_{i=1}^{n} X_{i}$; the sequence of random variables $\left\{S_{n}\right\}_{n \in \mathbb{N}}$ is a random walk on $\mathbb{Z}$. The piecewise linearization $\ell_{X}(t)$ of $X=\left(X_{1}, \ldots, X_{n}\right)$ is a piecewise linear function with breakpoints $k / n$, such that

$$
\ell_{X}\left(\frac{k}{n}\right)=\frac{S(k)}{\sqrt{n}} \quad(1 \leq k \leq n) .
$$

For a particular value $x \in\{ \pm 1\}^{n}$ of $X$, this agrees with our definition of $\ell_{x}$ in Section 1. Standard Brownian motion is a random function $W \in C[0,1]$. A good introductory reference is Durrett [7. There are several results to the effect that $\ell_{X}$ is an approximation of $W$, similar to how a finite binary sequence $A \uparrow n=$ $(A(0), \ldots, A(n-1))$ is an approximation of $A=(A(0), A(1), \ldots)$.

Suppose that one wants to define an i.i.d. sequence $X_{1}, X_{2}, \ldots$ of random variables with a given distribution so that the partial sums are as close to Brownian 
motion as possible. The result we need from Komlós, Major, and Tusnády [1, 12, is as follows.

Theorem 2.1. Assume that $\mathbb{E}\left(X_{k}\right)=0, \operatorname{Var}\left(X_{k}\right)=1$ and the moment generating function $\mathbb{E}\left(e^{u X_{k}}\right)<\infty$ for $|u| \leq u_{0}, u_{0}>0$. Let $S(k)=X_{1}+\cdots+X_{k}, k \geq 1$, be the partial sums. If Brownian motion $(W(t))_{t \geq 0}$ is given, then for any $n \geq 1$ there exists a sequence of transformations applied to $W(1), W(2), \ldots$ so that one obtains the desired partial sums $S(1), S(2), \ldots$ and the difference between the two sequences is the smallest possible:

$$
\mathbb{P}\left\{\max _{0 \leq k \leq n}|S(k)-W(k)|>C_{0} \log n+x\right\}<K_{0} e^{-\lambda x},
$$

for any $n \geq 1$ and $x>0$, where $C_{0}, K_{0}, \lambda$ are positive constants that may depend on the distribution of $X_{k}$, but not on $n$ or $x$. Moreover, $\lambda$ can be made arbitrarily large by choosing a large enough $C_{0}$.

Taking $x=C_{0} \log n$ in Theorem 2.1 one obtains

$$
\mathbb{P}\left\{\max _{0 \leq k \leq n}|S(k)-W(k)|>2 C_{0} \log n\right\}<K_{0} n^{-\lambda C_{0}},
$$

where $n \geq 1$ is arbitrary and one may assume that $\alpha:=\lambda C_{0} \geq 2$.

Lemma 2.2 (Brownian scaling relation, [7, p. 372]). Let $\alpha \geq 0$. For standard Brownian motion (with $W_{0}=0$ ), the stochastic process

$$
\left\{W_{s \alpha}\right\}_{s \geq 0}
$$

has the same distribution as

$$
\left\{\sqrt{\alpha} \cdot W_{s}\right\}_{s \geq 0} .
$$

Theorem 2.3. There exists Brownian motion $(W(t))_{t \geq 0}$, and for each $n \geq 1$, random variables $X_{k, n}$ for $1 \leq k \leq n$, all defined on the same probability space, such that for each $n \geq 1$,

1. the random variables $X_{1, n}, \ldots, X_{n, n}$ are mutually independent,

2. $\mathbb{P}\left\{X_{k, n}=1\right\}=\mathbb{P}\left\{X_{k, n}=-1\right\}=\frac{1}{2}$,

3. there are constants $c_{1}, c_{2}$, and $\alpha \geq 2$, such that if $X^{(n)}=\left(X_{1, n}, \ldots, X_{n, n}\right)$,

$$
\mathbb{P}\left\{\sup _{0 \leq t \leq 1}\left|W(t)-\ell_{X^{(n)}}(t)\right| \geq c_{1} \frac{\log n}{\sqrt{n}}\right\} \leq \frac{c_{2}}{n^{\alpha}} .
$$

Proof. By the self-similarity of Brownian motion (Lemma 2.2) defining

$$
W^{(n)}(t)=\sqrt{n} W\left(\frac{t}{n}\right)
$$

for each $n \geq 1, W^{(n)}$ is standard Brownian motion as well. Use $W^{(n)}$ in the KMT construction described above to obtain $X^{(n)}$. Then by (2.2),

$$
\mathbb{P}\left\{\max _{0 \leq k \leq n}\left|W\left(\frac{k}{n}\right)-\ell_{X^{(n)}}\left(\frac{k}{n}\right)\right|>2 C_{0} \frac{\log n}{\sqrt{n}}\right\}<K_{0} n^{-\alpha} .
$$

By Csörgö and Révész [6, Lemma 1.1.1] we have that for any $\epsilon>0$ there exists $C>0$ such that for any $n \geq 1$ and $c \geq((\alpha+1)(2+\varepsilon))^{1 / 2}$, one has

$$
\mathbb{P}\left\{\sup _{0 \leq k<n, 0 \leq t-\frac{k}{n} \leq \frac{1}{n}}\left|W(t)-W\left(\frac{k}{n}\right)\right| \geq c\left(\frac{\log n}{n}\right)^{\frac{1}{2}}\right\} \leq C n^{1-\frac{c^{2}}{2+\epsilon}} \leq C n^{-\alpha}
$$


Finally, by its definition,

$$
\sup _{0 \leq k<n, 0 \leq t-\frac{k}{n} \leq \frac{1}{n}}\left|\ell_{X^{(n)}}(t)-\ell_{X^{(n)}}\left(\frac{k}{n}\right)\right| \leq \frac{1}{\sqrt{n}} .
$$

(2.3), (2.4), and (2.5) together prove the theorem.

Corollary 2.4. We can assert furthermore that for each $N \geq 1$,

$$
\mathbb{P}\left(\bigcup_{n>N}\left\{d\left(W, \ell_{X^{(n)}}\right) \geq c \frac{\log n}{\sqrt{n}}\right\}\right) \leq \frac{c_{2}}{N} .
$$

Proof. By Theorem 2.3.

$$
\mathbb{P}\left(\bigcup_{n>N}\left\{d\left(W, \ell_{X(n)}\right) \geq c \frac{\log n}{\sqrt{n}}\right\}\right) \leq \sum_{n>N} \frac{c_{2}}{n^{\alpha}} \leq \frac{c_{2}}{N} .
$$

\section{Application to Kolmogorov complexity}

The following lemma is a variation on a well-known fact about Kolmogorov complexity.

Lemma 3.1. If $\mathbb{P}$ is a distribution on sequences

$$
\left\{x_{n}\right\}_{n \in \mathbb{N}} \in \prod_{n \in \mathbb{N}}\{ \pm 1\}^{n}=\{ \pm 1\}^{0} \times\{ \pm 1\}^{1} \times \cdots
$$

such that the marginal distribution of each $x_{n}$ is uniform on $\{ \pm 1\}^{n}$, then

$$
\mathbb{P}\left(\exists b \forall n K\left(x_{n}\right) \geq n-b\right)=1 .
$$

Proof. Using $\sum_{\text {all } \sigma} 2^{-K(\sigma)} \leq \sum_{p \text { halts }} 2^{-|p|} \leq 1$, we have

$$
\begin{aligned}
\mathbb{P}\left(\exists n K\left(x_{n}\right)<n-b\right) & \leq \sum_{\{\sigma: K(\sigma)<|\sigma|-b\}} \mathbb{P}\left(x_{|\sigma|}=\sigma\right)=\sum_{\{\sigma: K(\sigma)<|\sigma|-b\}} 2^{-|\sigma|} \\
& \leq \sum_{\text {all } \sigma} 2^{-(K(\sigma)+b)} \leq 2^{-b} .
\end{aligned}
$$

We are now ready to show that Asarin's Theorem holds with the bound $n^{-1 / 10}$ replaced by $O(\log n / \sqrt{n})$.

Theorem 3.2. $W \in C[0,1]$ is a Martin-Löf random Brownian motion if and only if there are constants $b, c$ such that for all but finitely many $n \in \mathbb{N}$ there is a string $x=\left(x_{1}, \ldots, x_{n}\right) \in\{ \pm 1\}^{n}$ such that

$$
d\left(\ell_{x}, W\right) \leq \frac{c \log n}{\sqrt{n}} \quad \text { and } \quad K\left(x_{1}, \ldots, x_{n}\right) \geq n-b .
$$

Proof. In light of Asarin's Theorem, Theorem 1.4, it suffices to show the only if direction. For $b \in \mathbb{N}$, let

$$
U_{b}=\bigcup_{n>b} \bigcap_{x \in\{ \pm 1\}^{n}}\left\{W:\left(d\left(\ell_{x}, W\right) \leq \frac{c \log n}{\sqrt{n}}\right) \rightarrow(K(x)<n-b)\right\} .
$$

We verify that $U_{b}$ is a $\Sigma_{1}^{0}$ class: 
(i) $d\left(\ell_{x}, W\right)>c \log n / \sqrt{n}$ holds if and only if $W$ belongs to some $T_{n}$ such that all the members $B$ of $T_{n}$ satisfy $d\left(\ell_{x}, B\right)>c \log n / \sqrt{n}$, and

(ii) the property $K(x)<n-b$ asserts that a short description of $x$ exists.

Let $X^{(n)}$ be a random variable as in Theorem 2.3 , and let

$$
V_{b}=\bigcup_{n>b}\left\{W:\left(d\left(\ell_{X^{(n)}}, W\right) \leq \frac{c \log n}{\sqrt{n}}\right) \rightarrow\left(K\left(X^{(n)}\right)<n-b\right)\right\} .
$$

Note that $V_{b}$ is a random set, i.e. it is itself a random variable, but we will only use the following two auxiliary properties of $V_{b}: V_{b}$ always contains the deterministic set $U_{b}$, and $V_{b}$ has small Wiener measure. Indeed, by Corollary 2.4 ,

$$
\mathbb{P}\left(\bigcup_{n>b}\left\{W: d\left(\ell_{X^{(n)}}, W\right)>\frac{c \log n}{\sqrt{n}}\right\}\right) \leq \frac{c_{2}}{b},
$$

and by Lemma 3.1 ,

$$
\mathbb{P}\left((\exists n) K\left(X^{(n)}\right)<n-b\right) \leq 2^{-b},
$$

SO

$$
\mathbb{P}\left(U_{b}\right) \leq \mathbb{P}\left(V_{b}\right) \leq \frac{c_{2}}{b}+2^{-b},
$$

since clearly $U_{b} \subseteq V_{b}$. Thus if $W$ is Martin-Löf random, then there is some $b$ such that for all $n>\bar{b}$, there is some $x=\left(x_{1}, \ldots, x_{n}\right)$ with $\ell_{x}(t)$ that lies within $\frac{c \log n}{\sqrt{n}}$ of $W(t)$ and $K(x) \geq n-b$.

Remark 3.3. A complementary result to Theorem 3.2 was obtained by Fouché [9. Theorem 5], who showed that from the first $n$ bits of a Martin-Löf random real $A$ one can uniformly compute a finite linear combination of piecewise linear functions (of a different type from the ones considered here) that lies within $O(\log n / \sqrt{n}$ ) of an associated Martin-Löf random path of Brownian motion $W_{A}$.

\section{A limitation on FURther improvements}

The rate $O(\log n / \sqrt{n})$ in our improved version of Asarin's Theorem, Theorem 3.2 , cannot be further improved to $\frac{1}{2} \sqrt{\frac{\log n}{n}}$. We now prove this by using Lévy's analysis of the modulus of continuity of Brownian motion. A function $g:[0,1] \rightarrow \mathbb{R}$ is Hölder continuous of order $\gamma$ if there is a constant $C$ such that for all $x, y \in[0,1],|f(x)-f(y)| \leq C|x-y|^{\gamma}$. Wiener [18] showed that Brownian motion is almost surely Hölder continuous of any order $\gamma<1 / 2$, but this does not extend to order $\gamma=1 / 2$ (see for example Durrett [7, Exercise 2.4, p. 382]), and Lévy obtained even more precise information.

Definition 4.1 (Lévy's modulus of continuity). For a path of Brownian motion $W$, let

$$
\operatorname{osc}(\delta)=\sup \{|W(s)-W(t)|: s, t \in[0,1],|t-s|<\delta\} .
$$

Theorem 4.2 (Lévy [14]; see Durrett [7, p. 394]). Almost surely,

$$
\limsup _{\delta \rightarrow 0} \operatorname{osc}(\delta) /(\delta \log (1 / \delta))^{1 / 2}=\sqrt{2}
$$


Thus, we will give a lower bound for possible rates in Asarin's Theorem by using the fact that a typical path of Brownian motion should have an increment $|W(s)-W(t)| \approx n^{-1 / 2} \sqrt{\log n}$ somewhere in an interval of length $1 / n$, while an increment $\left|\ell_{x^{(n)}}(s)-\ell_{x^{(n)}}(t)\right|$ of an approximating broken line is only $n^{-1 / 2}$ on the same interval.

Corollary 4.3. Almost surely, for each $\theta>0$ and $\varepsilon>0$ there exists $0<\delta<\varepsilon$ and $s, t \in[0,1]$ with $|t-s|<\delta$ such that

$$
|W(s)-W(t)| \geq(\sqrt{2}-\theta)(\delta \log (1 / \delta))^{1 / 2} .
$$

Proof. By Theorem 4.2,

$$
\lim _{\varepsilon \rightarrow 0} \sup _{0<\delta<\varepsilon} \operatorname{osc}(\delta) /(\delta \log (1 / \delta))^{1 / 2} \geq \sqrt{2},
$$

so for each $\varepsilon>0$ and $\theta>0$ there exists $0<\delta<\varepsilon$ such that

$$
\operatorname{osc}(\delta) /(\delta \log (1 / \delta))^{1 / 2} \geq \sqrt{2}-\theta .
$$

Thus there exists $s, t \in[0,1]$ with $|t-s|<\delta$ and

$$
|W(s)-W(t)| \geq(\sqrt{2}-\theta)(\delta \log (1 / \delta))^{1 / 2} .
$$

Corollary 4.4. Almost surely for Brownian motion $W$, there is no $n_{W}$ such that for all $n \geq n_{W}$ there is an $x \in\{ \pm 1\}^{n}$ with

$$
d\left(\ell_{x}, W\right) \leq \frac{1}{2} \sqrt{\frac{\log n}{n}} .
$$

Proof. Suppose otherwise; i.e., with positive probability there is an $n_{W}$ such that for all $n \geq n_{W}$, there is a length $n$ walk $g$ within $f(n):=\frac{1}{2} \sqrt{\frac{\log n}{n}}$ of $W$.

Let $0<\gamma<1 / 2$. Since $\gamma>0$, there is a $\theta>0$ such that

$$
2-\gamma \leq\left(\frac{\sqrt{2}-\theta}{1+\theta}\right)^{2}
$$

Let $\varepsilon_{W}>0$ be small enough to guarantee that if $0<\delta \leq \delta^{\prime}<\varepsilon_{W}$ and $\frac{1}{n+1}<\delta$, then

(1) $(\delta \log (1 / \delta))^{1 / 2} \leq\left(\delta^{\prime} \log \left(1 / \delta^{\prime}\right)\right)^{1 / 2}$,

(2) $n \geq n_{W}$,

(3) $1 \leq \theta \sqrt{\log n}$, and

(4) $n \geq 2$.

By Corollary 4.3. for almost all $W$ we have that for all $\varepsilon>0$, in particular for $\varepsilon=\varepsilon_{W}$ when $\varepsilon_{W}$ exists, there exist $0<\delta<\varepsilon$ and $s, t \in[0,1]$ so that

$$
|s-t|<\delta \quad \text { and } \quad(\sqrt{2}-\theta) \sqrt{\delta \log (1 / \delta)} \leq|W(t)-W(s)| .
$$

Let $n$ be such that $\frac{1}{n+1}<\delta<\frac{1}{n}$. Then for such $s, t$, using (1), (2), and (3),

$$
\begin{gathered}
(\sqrt{2}-\theta) \sqrt{\frac{\log (n+1)}{n+1}} \leq(\sqrt{2}-\theta) \sqrt{\delta \log (1 / \delta)} \leq|W(t)-W(s)| \\
\leq|W(t)-g(t)|+|g(t)-g(s)|+|g(s)-W(s)| \leq 2 f(n)+n^{-1 / 2} \leq(1+\theta) \sqrt{\frac{\log n}{n}}
\end{gathered}
$$


and hence using (4),

$$
2-\gamma \leq\left(\frac{\sqrt{2}-\theta}{1+\theta}\right)^{2} \leq \frac{\log n}{\log (n+1)} \cdot \frac{n+1}{n} \leq \frac{3}{2},
$$

which contradicts $\gamma<1 / 2$.

We deduce that Asarin's Theorem, Theorem 1.4 does not hold for the bound $\frac{1}{2} \sqrt{\frac{\log n}{n}}$.

Corollary 4.5. The assumption that $W \in C[0,1]$ is a Martin-Löf random Brownian motion does not imply that for all but finitely many $n \in \mathbb{N}$ there is any string $x=\left(x_{1}, \ldots, x_{n}\right) \in\{ \pm 1\}^{n}$ such that

$$
d\left(\ell_{x}, W\right) \leq \frac{1}{2} \sqrt{\frac{\log n}{n}}
$$

at all (let alone such a string with $K\left(x_{1}, \ldots, x_{n}\right) \geq n-c$ ).

Proof. The set of Martin-Löf random Brownian paths has positive measure (in fact measure 1), and so we would have a contradiction to Corollary 4.4.

\section{FUTURE WORK}

\subsection{Further narrowing of the bounds.}

Question 5.1. Does Asarin's Theorem hold for a rate $r(n)=o(\log n / \sqrt{n})$ ?

If the answer to Question 5.1 is yes, then the ball of radius $r(n)$ around $W$ is "typical" enough to contain $\ell_{x}$ for a string $x \in\{ \pm 1\}^{n}$ with $K(x) \geq^{+} n$. If we then consider the random variable $X$ that selects (uniformly) a string $x \in\{ \pm 1\}^{n}$ with $d\left(W, \ell_{x}\right) \leq r(n)$, we might suspect that $X$ would be (at least approximately) uniformly distributed on $\{ \pm 1\}^{n}$ (for all but finitely many $n$ ). That $X$ could not literally be uniformly distributed is a consequence of the following result.

Theorem 5.2. Let $X_{k, n}(k=1, \ldots, n ; n=1,2, \ldots)$ be a triangular array of identically distributed random variables with zero expectation and unit variance such that $X_{1, n}, \ldots, X_{n, n}$ are mutually independent. Let $S^{(n)}(k)=X_{1, n}+\cdots+X_{k, n}$ and $W^{(n)}(t)(t \geq 0 ; n=1,2, \ldots)$ be a sequence of Brownian motions such that

$$
\max _{1 \leq k \leq n}\left|S^{(n)}(k)-W^{(n)}(k)\right|=o(\log n) \quad \text { (a.s.). }
$$

Then the distribution of $X_{k, n}$ is standard normal.

Proof. This theorem follows easily from Csörgö and Révész [6, Theorem 2.3.2 $]^{2}$ if the i.i.d. sequence $X_{n}$ there is replaced by a triangular array $X_{k, n}$. The underlying Theorems 2.3.1, 2.4.3-2.4.5 there can be modified accordingly as well.

\footnotetext{
${ }^{2}$ This result of Csörgő and Révész was strengthened by Bass and Burdzy [3] Theorem 5.6].
} 
5.2. Rate distortion theory. We are grateful to the referee for the following interesting problem.

Question 5.3. What is the complexity of the simplest walk within $O(\log n / \sqrt{n})$ of the Brownian path?

In the framework of classical rate distortion theory, this asks how low the complexity of a walk can be if we still want to be able to reconstruct a reasonable approximation to the Brownian path from it. Question 5.3 appears to be related to the question of how many walks are close to the Brownian path, i.e., the size of balls

$$
B_{\varepsilon}(W)=\left\{x \in\{ \pm 1\}^{n}: d\left(\ell_{x}, W\right) \leq \varepsilon\right\}
$$

centered at the Brownian path. Chen [5, Theorem 2] obtained precise information about the size of balls centered at the constant function 0. His work is not immediately applicable here, however, because by Corollary 4.4 there will be choices of $\varepsilon$ where $B_{\varepsilon}(W)=\varnothing$, while $B_{\varepsilon}(0)$ is fairly large.

5.3. Schnorr randomness. While the most studied form of algorithmic randomness is Martin-Löf randomness, there are other variants such as Schnorr randomness. Here tests are required to have the probability of $U_{n}$ equal to $2^{-n}$, or equivalently any computable function of $n$ that goes effectively to zero. Schnorr randomness is preferable in the sense that if a finite number of almost sure properties is shown to hold for each Schnorr random function, then there is in fact a computable function displaying this almost sure behavior. It would be somewhat disturbing to prove a law without being able to provide a computable example illustrating it. Fortunately, most laws encountered in practice hold for all Schnorr random functions.

Question 5.4. Is there an analogous result to our main result, Theorem 3.2, for Schnorr random Brownian motion?

In one direction, the problem here is to compute the measure of analogues of the sets $U_{n}$. We suspect that this might be possible by analyzing the speed of convergence in Donsker's theorem.

\section{REFERENCES}

[1] E. A. Asarin, Individual random signals: an approach based on complexity, doctoral dissertation, Moscow State University, 1988.

[2] E. A. Asarin and A. V. Pokrovskiǔ, Application of Kolmogorov complexity to the analysis of the dynamics of controllable systems, Avtomat. i Telemekh. 1 (1986), 25-33 (Russian, with English summary). Automat. Remote Control 47 (1986), no. 1, part 1, 21-28. MR831773 (87e:93096)

[3] Richard F. Bass and Krzysztof Burdzy, Stochastic bifurcation models, Ann. Probab. 27 (1999), no. 1, 50-108. MR.1681142 (2000b:60201)

[4] Gregory J. Chaitin, A theory of program size formally identical to information theory, J. Assoc. Comput. Mach. 22 (1975), 329-340. MR0411829 (53:15557)

[5] Xia Chen, Moderate and small deviations for the ranges of one-dimensional random walks, J. Theoret. Probab. 19 (2006), no. 3, 721-739. MR2280517 (2007k:60075)

[6] M. Csörgő and P. Révész, Strong approximations in probability and statistics, Probability and Mathematical Statistics, Academic Press, Inc. [Harcourt Brace Jovanovich Publishers], New York, 1981. MR666546 (84d:60050)

[7] Richard Durrett, Probability: Theory and examples, 2nd ed., Duxbury Press, Belmont, CA, 1996. MR1609153 (98m:60001) 
[8] Willem Fouché, Arithmetical representations of Brownian motion. I, J. Symbolic Logic 65 (2000), no. 1, 421-442. MR.1782129 (2002b:68038)

[9] Willem L. Fouché, The descriptive complexity of Brownian motion, Adv. Math. 155 (2000), no. 2, 317-343. MR.1794714 (2002e:68044)

[10] Péter Gács, Exact expressions for some randomness tests, Z. Math. Logik Grundlag. Math. 26 (1980), no. 5, 385-394. MR589329 (82c:65004)

[11] J. Komlós, P. Major, and G. Tusnády, An approximation of partial sums of independent RV's and the sample DF. I, Z. Wahrscheinlichkeitstheorie und Verw. Gebiete 32 (1975), 111-131. MR0375412(51:11605b)

$[12] \_$An approximation of partial sums of independent RV's, and the sample DF. II, Z. Wahrscheinlichkeitstheorie und Verw. Gebiete 34 (1976), no. 1, 33-58. MR0402883 (53:6697)

[13] Bjørn Kjos-Hanssen and Anil Nerode, Effective dimension of points visited by Brownian motion, Theoret. Comput. Sci. 410 (2009), no. 4-5, 347-354. MR.2493984 (2009k:68100)

[14] P. Lévy, Théorie de l'addition des variables aléatoires, 2nd ed., Gauthier-Villars, Paris, 1954.

[15] Ming Li and Paul Vitányi, An introduction to Kolmogorov complexity and its applications, 3rd ed., Texts in Computer Science, Springer, New York, 2008. MR2494387 (2010c:68058)

[16] André Nies, Computability and randomness, Oxford Logic Guides, vol. 51, Oxford University Press, Oxford, 2009. MR.2548883

[17] Claus-Peter Schnorr, Zufälligkeit und Wahrscheinlichkeit. Eine algorithmische Begründung der Wahrscheinlichkeitstheorie, Lecture Notes in Mathematics, Vol. 218, Springer-Verlag, Berlin, 1971. MR0414225 (54:2328)

[18] Norbert Wiener, Differential space, J. Math. Phys. 2 (1923), 131-174.

Department of Mathematics, University of HaWai'i at MĀnoa, 2565 McCarthy Mall, Honolulu, HaWail 96822

E-mail address: bjoern@math.hawaii.edu

Department of Mathematics, Budapest University of Technology and Economics, BudAPEST, Hungary

E-mail address: szabados@math.bme.hu 